\title{
Spitzer Warm Mission Workshop Introduction
}

\author{
Lisa J. Storrie-Lombardi and Sean Carey \\ Spitzer Science Center, California Institute of Technology, MS314-6, Pasadena, CA 91125 USA
}

\begin{abstract}
.
The Spitzer Warm Mission Workshop was held June 4-5, 2007, to explore the science drivers for the warm Spitzer mission and help the Spitzer Science Center develop a new science operations philosophy. We must continue to maximize the science return with the reduced resources available, both using (a) the shortest two IRAC channels, and (b) archival research with the rich Spitzer archive. This paper summarizes the overview slides presented to the workshop participants.
\end{abstract}

Keywords: Spitzer Space Telescope

PACS: $98.85 . \mathrm{Hp}$

\section{INTRODUCTION}

\subsection{Workshop Format}

\section{Monday}

- Introduction to the Warm Mission

- Overview

- Mission plans and questions

- Solicited white paper reports

- Contributed white paper summaries

- Splitzer group discussion

- Solar Systems

- Our Galaxy

- Nearby Galaxies

- Distant Galaxies

\section{Tuesday}

- Archive Presentation

- Splinter group summaries

- Discussion 


\subsection{Workshop Steering Committee}

Chair

Nearby Universe

Extrasolar Planets

Stars, Brown dwarfs

Solar System

Galactic Structure and ISM

Star Formation

Distant Universe
Pat McCarthy (OCIW)

Daniela Calzetti (U. Mass)

Drake Deming (GSFC)

Jill Knapp (Princeton)

Carey Lisse (JHU-APL)

Mike Skrutskie (U. Virginia)

Steve Strom (NOAO)

Pieter van Dokkum (Yale)

\section{OVERVIEW}

\subsection{Life After Helium}

- Observatory has ample reserves on consumables, power, etc.

- Cryo-telescope assembly expected to equilibrate at $\sim 25-29 \mathrm{~K}$

- IRAC will have essentially unchanged sensitivity at 3.6 and $4.5 \mu \mathrm{m}$

- All other detectors non-operational

- Spitzer archive will still be brimming with data

- Community will be in the first round of extracting science from the archive

\subsection{Our Vision: To Fully Exploit NASA's and the Community's Investment in the Spitzer Mission}

We will do this by:

- Capturing the full legacy of Spitzer into a robust, permanent archive.

- Expanding the science from Spitzer beyond the Liquid Helium lifetime through a vigorous archival research program.

- Utilizing the continuing observatory capabilities for unique, vital science possible only with Spitzer.

\section{The Data Archive}

- At the end of the Spitzer cryo-mission, we must reprocess the full data set to uniform calibration and minimal artifacts.

- Will leave a legacy for science utilization that will remain vital for decades.

- Will apply the full knowledge and understanding of Spitzer.

- Usefulness of Spitzer and return on investment will be enhanced by new generations of users.

- Exact contents will depend on resources (therefore NASA environment), and community needs and inputs. 


\section{Exploiting the Spitzer Data Archive: Community Support}

- Quality and uniformity are critical for new science leveraging the entire archive.

- Optimized calibration, minimized artifacts in the final processing.

- Full realization of the science potential of the permanent archive requires:

- Adequate funding to the science community.

- Support by active scientists at SSC, providing expertise and adapting software.

- Without a dedicated support plan, archival research funding would be available only through ADP, and technical support at the SSC would be minimal.

- ADP funding was $\$ 2$ million in 2004 for $\sim 30$ mission data sets

- As currently established the ADP funding is inadequate to support a meaningful Spitzer archival program

\section{Warm Spitzer: A Unique Asset}

- At end of cryogenic phase, Spitzer will still be a unique space observatory

- Telescope should equilibrate at $<30 \mathrm{~K}$ in solar orbit.

- IRAC $5^{\prime} \times 5^{\prime}$ FOVs at 3.6 and $4.5 \mu \mathrm{m}$ will operate in parallel.

- 3 - $5 \mu \mathrm{m}$ sensitivity essentially unchanged from cryogenic phase, unmatched until JWST flies

- No measurable degradation in the IRAC arrays to this point.

- Observatory represents over a billion dollars cumulative investment.

- Powerful capabilities

- Finely tuned, calibrated science instrument.

- Wide-field, superb mapping engine.

- Time-domain access on all scales from milli-seconds to years.

- Well-honed operations

- 6 years of experience and optimal efficiency.

- Stable, efficient ground support and data analy sis system.

\section{Warm Spitzer Sensitivity - Figure 1}

- IRAC 3.6 and $4.5 \mu \mathrm{m}$ bands match WISE bands 1 and 2 and lie in the JWST sweet spot.

- $\sim 3$ orders of magnitude between WISE and JWST sensitivity will be the domain of warm Spitzer/IRAC as the tool of choice.

- Shallow integrations can follow-up on WISE discoveries.

- Deepest integrations will provide path-finding science for JWST. 


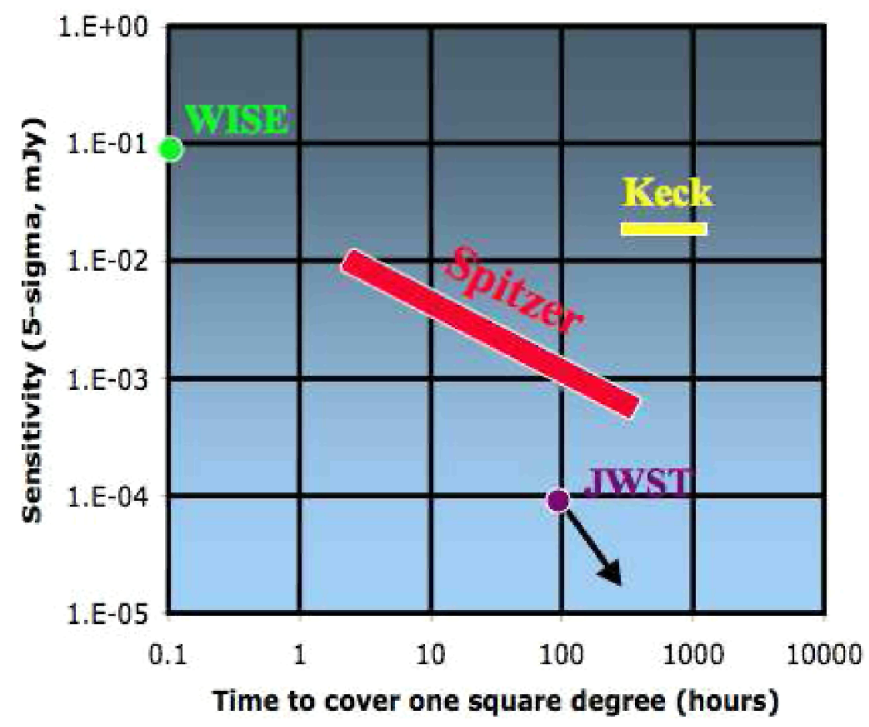

FIGURE 1. Spitzer sen sitivity during the warm mission phase compared to predicted WISE and JWST performance and measured mid-IR performance with Keck. This figure is adapted from the Spitzer-WISE memo at http://ssc.spitzer.caltech.edu/documents/wisememo.doc.pdf.

\section{IRAC PERFORMANCE AND OPERATIONS IN THE WARM SPITZER MISSION}

\section{Operating Environment Assumptions}

- Spitzer will be passively cooled after cryogen runs out.

- Telescope $\sim 24-25 \mathrm{~K}$

- Multiple Instrument chamber (IRAC) $\sim 25-29 \mathrm{~K}$

- Warm up above MIPS, IRS and 5.8 and $8.0 \mu \mathrm{m}$ operating temperatures occurs within 12 hours of cryogen running out.

- Telescope temperature equilibrium occurs within 4 weeks.

- OPZ (operational pointing zone) remains the same.

- Same effective downlink rate as cryogenic operations.

- IRAC data rate is halved.

- Pointing system exhibits same stability and accuracy.

\section{Predicted IRAC Performance}

- Observations with 3.6 and $4.5 \mu \mathrm{m}$ (InSb) arrays only.

- Temperature of arrays actively controlled.

- Arrays heated to operating temperature of $30 \mathrm{~K}$.

- Testing of similar arrays at $30 \mathrm{~K}$ at University of Rochester 


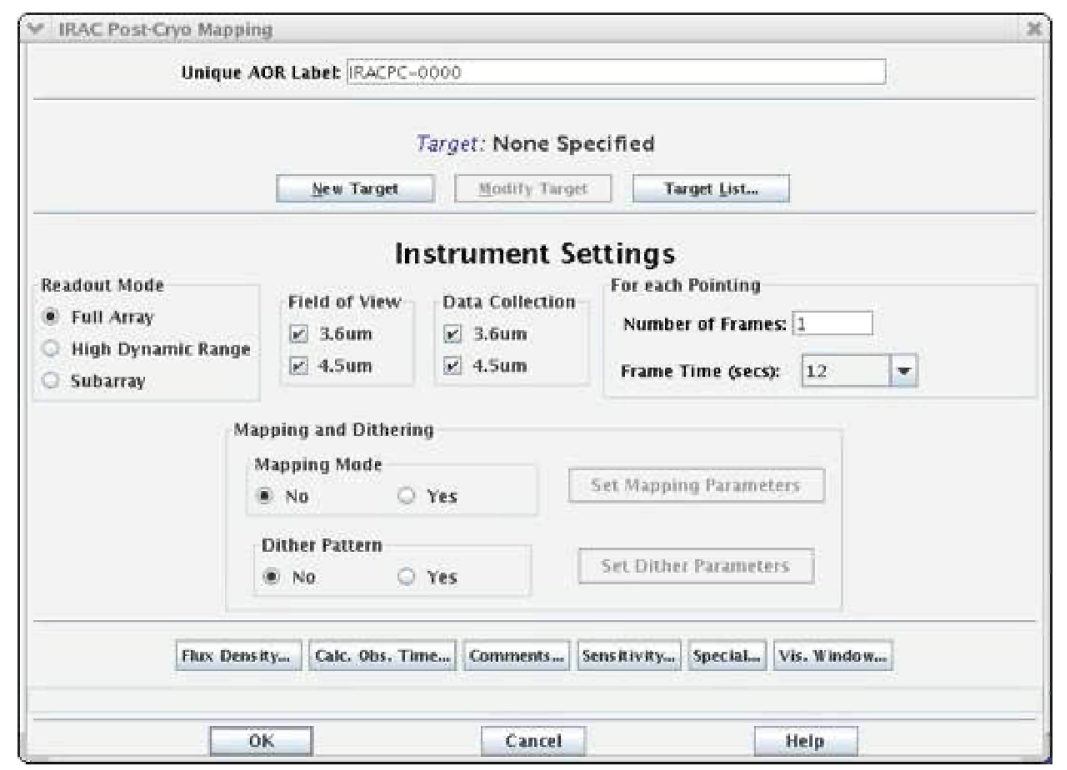

FIGURE 2. The warm mission IRAC AOT is shown in this screen shot from Spot. It operates similarly to the IRAC AOT in Spot today.

- No significant increase in dark current

- Slight increase in read noise

- Most observations should still be background/photon dominated

- For frame times $>30$ seconds, arrays should be background limited for all backgrounds

- Latents could be more significant but possibly decay faster

- Redundancy will be important

\section{Warm IRAC Astronomical Observing Template (AOT) - Figure 2}

- Data taking at 3.6 and $4.5 \mu \mathrm{m}$ only.

- Can choose to take data in only one channel to reduce data volume.

- Dither and mapping options remain the same.

- Full frame mode

$-0.4^{*}, 2,6^{*}, 12,30,100,200,400^{*}$ second frame times $\left({ }^{*}\right.$ potential new frame times)

- Use of 200 and 400 second frame times contingent on improved noise properties for deep images

- High Dynamic Range mode

$-12,30,100,200,400^{*}$ second frame times

- Subarray mode

$-0.02,0.1,0.4$ second frame times 


\section{Warm Instrument Characterization}

- First month of the warm mission

- Sample, simple science programs during weeks 2-4 to fill gaps during functional observations

- Week 1 - Functional checkout

- Aliveness test, Determine temperature set points, optimize array biases

- Week 2 - Array properties

- First month of the warm mission

- Set Fowler sampling and finalize frame times

- Calculate noise properties

- Latent characterization

- AOT checkout

- Week 3 - Baseline calibrations

- Dark and Flat calibrations

- Stellar calibrations

- Focus check

- Distortion map

- PRF measurement

- Week 4 - Science Verifications

- Deep image

- Photometric monitoring

- Galactic shallow survey

\section{MISSION PLANS}

- Pre-launch mission plan

-5 to $5-1 / 2$ year cryogenic mission

$-1-1 / 2$ to 2 year warm mission

- 1 year close-out

- Current Proposal

$-5-1 / 2$ year cryogenic mission

- 5 year warm mission

- 1 year close-out

- Bottom Line

- $\$ 110$ million for 3 additional years of warm observing operations

- \$50 million for operations $+\$ 60$ million for user community 


\section{OPERATIONAL ASPECTS}

- Expect high observing efficiency to continue

- Execute $6500-7000$ hours of science per year

- The challenge is to conduct this mission cost-effectively: maximize the science to cost ratio.

- Planning is based on model of half current staff at SSC, JPL and LMA when final reprocessing of cryo-mission data is complete

- To operate mission with this work-force requires substantial simplifications of operations

* Substantially reduced number of supported programs

* Simplification of planning and scheduling

* Fewer scheduling interrupts

* Reduced engineering staff for performance analysis and anomaly response

- Look for economies of scale without sacrificing the science

- Maintain peer-review process

* Make it less expensive

* Annual review costs $\$ 250 \mathrm{k}$ not counting the FTEs supporting it

- Shift emphasis to large and huge programs, since well have 7000 hours per year to allocate

- Fund data analysis and archival research at an appropriate level

- Streamline science and mission operations to the max

- Engage the community in the planning process - You are Here!

\section{COMMUNITY SUPPORT}

Continue providing substantial support for the community.

- Currently send \$30-35 million year to the User Community

GO-Legacy

Archive-Theory

GTO science funding

Fellowship program

Overhead

Spitzer constant ( $\$$ per hour) $\sim \$ 3 k$
\$18-22 million

$\$ 2$ million $(\$ 2.7$ in Cycle-4)

$\sim \$ 7$ million

$\sim \$ 1.6$ million

$\$ 3$ million $(\sim 10 \%)$

- Warm Mission Plan - \$20 million per year to User Community

Warm Observing

Archive/Theory

Fellowship program

Overhead

Spitzer constant (\$ per hour) $\sim \$ 1.8 k$
$\$ 13$ million

$\$ 4.8$ million

$\$ 1$ million

$\$ 1.2$ million $(\sim 6 \%)$ 


\section{STRAWMAN PLANS FOR DISCUSSION AT THE WORKSHOP}

\subsection{Transition to the Warm Mission}

- Best estimate for cryogen depletion is March-April 2009.

- We must have $\sim 1000$ hours ready to execute by February 2009

- Program selected and advertised in advance based on input from Workshop or separate selection process

- Execute in HDF style

- Observations designed by science committee (External+SSC)

- No proprietary period

- No direct funding

- Archival funding available via regular review process

\subsection{Observing Proposals}

\section{Proposal Categories - Observing}

- Small $<100$ hours (Should this be 50 hours?)

- AORs required

- 1-year proprietary period

- 1000 hours maximum per cycle (2175 hours awarded in Cycle-4)

- Directors time (5-10\%)

* Could this be used for small category? $(<10 \mathrm{hrs}$ ?)

- Medium $100-500$ hours

- Large 500-2000 hours

- Huge $>2000$ hours

- Big programs

- Template AORs with proposals

- No proprietary period

- Really big programs could be executed over 2 years

- No direct funding for proposals $<10$ hours

- Page charges for successful Spitzer proposers paid directly by the SSC?

\subsection{Archive-Theory Proposals}

- Continue to offer 1-year Archive-Theory proposals ( 50-100k)

- Legacy Archive

- Multi-year archive programs

- Return enhanced data product deliverables to SSC-IRSA 
- Up to $\$ 500 \mathrm{k}$

- Large Archive

- Multi-year archive programs

- No enhanced data product deliverables

- Up to $\$ 300 \mathrm{k}$

- Multi-year Theory - should we support these too?

-What fraction of the total community funding should go to Archive/Theory?

- Should the amount for each category be preordained?

\subsection{Review Process}

- Hold annual proposal calls and review meeting

- Does the process need to be annual (would 18 month centers do?)

- Do the review process in two phases

- Save \$200k per year on review costs = one FTE

- Phase 1: remote review of all proposals and submission of grades

* Top $10 \%$ of small proposals awarded time?

* Additional small allocation determined by lottery from proposals ranked $10-\mathrm{XX} \%$

* Top $20-25 \%$ (or highest ranked 1000 hours) of small proposals awarded time?

* Big programs (medium, large and huge) forwarded to TAC to provide oversubscription factor of 2

- Phase 2: face-to-face meeting of TAC to select big programs

- Archive-Theory-Observing all reviewed together

- Variant:

- Review Archive-Theory six months out of phase with observing

- Same review panels and TAC

- TAC meets remotely to select Legacy archive programs

\subsection{Program Support}

- Program reviews

- Cursory technical checks

- No duplication checks after selection

- Scheduling

- Continue to schedule in weekly blocks

- 24-36 hr PAOs (periods of autonomous operations)

- Low impact ToOs - no restrictions

- Select one-high impact ToO/year (currently we select up to 10) 
- Archive/Data Rights

- No embargo checking for large, public surveys

- Advertise this in Cycle-5 as it may impact those programs

\section{SUMMARY OF QUESTIONS FOR THE WORKSHOP}

- What are the most important science drivers for a warm Spitzer mission?

- What should be the duration of the warm mission?

- What public HDF-style program should be prepared for the cryo/warm transition period?

- What is the appropriate balance between smaller and larger programs?

- Are ToOs an important component of the warm mission? If yes, at what level?

- Should any science programs be specifically solicited for the warm mission?

- Are there any huge ( $>5000$ hours) projects that should be done? If yes, how should they be selected and organized?

- How does the community participate in science of big projects if not part of the executing teams?

- Can most of the review process be done remotely instead of bringing 100 people to Pasadena annually for a week?

- Should the review of observing proposals and archival-theory proposals be held at the same time or 6 months out of phase?

- Warm Transition Program

- Should specific enhanced data products be produced by SSC?

- Should we carve out a specific dollar amount to support archival research with these data?

- Observing Proposals

- What hour range should be defined as small? .. $<50 ?<100$ ?

- Use DDT for small category?

- No direct funding for very small programs?

- Do we have the right breakdown in categories?

- Should there be a preordained distribution of time between categories?

- Archive/Theory Proposals

- What fraction of the total community funding should go to Archive-Theory?

- Should the amount for each category be preordained?

- Review Process

- How often do we need to select programs?

- Should we use a lottery element for any of it? 


\section{ACKNOWLEDGMENTS}

The Spitzer Science Center would like to thank everyone who participated in the workshop and the preparation of the white papers. We greatly value the input we have received from the community. 